\title{
NONCOMMUTATIVE EXTENSIONS OF HILBERT RINGS
}

\section{CHARLES W. CURTIS}

1. Introduction. The main results in this paper concern the ideal theory and representations of a ring $S$ containing a ring $R$ in its center such that $S$ is a finitely generated $R$-module. If $R$ is a local ring, then the structure of $S$ has been investigated by Azumaya [1],1 who proved an extension of the Wedderburn-Malcev theorem for finitedimensional algebras. We impose the condition that $R$ be a Hilbert ring in the sense of Goldman [6], that is, in every homomorphic image of $R$, the nil radical and the Jacobson radical coincide. Then it is proved that in every homomorphic image of $S$, the lower radical and the Jacobson radical coincide. This result is a corollary of Theorem 4.3, which states that every prime ideal in $S$ is an intersection of primitive ideals. If $R$ contains a field $K$, then every homomorphic image of $R$ contains a subfield isomorphic to $K$, which we shall identify with $K$. If every field which is a homomorphic image of $R$ is a finite algebraic extension of $K$, then every irreducible representation of $S$ maps $S$ onto a finite-dimensional simple algebra over $K$, and the degrees of the absolutely irreducible representations of $S$ are bounded. Most of the theorems in the paper are based upon the simple fact that if $S$ satisfies the ascending chain condition for $R$-submodules, then every primitive ideal in $S$ contracts to a maximal ideal in $R$. Indeed, this theorem is valid under slightly weaker restrictions on $S$, but we shall postpone their discussion until $\S 3$.

In $\S 5$, we apply our results to the universal associative algebra $A$ of an arbitrary Lie algebra $L$ over a field $K$ of prime characteristic. We base our discussion upon a recent paper of N. Jacobson [10], in which it is proved that there exist elements $y_{1}, \cdots, y_{n}$ contained in the center $C$ of $A$, such that $A$ is a finitely generated module over $R=K\left[y_{1}, \cdots, y_{n}\right]$. Then $R$ is a Hilbert ring, and Theorems 4.2 and 4.3 are applicable to $A$. Since $A$ is semi-simple, $A$ has sufficiently many finite-dimensional irreducible representations, and from this fact we obtain the theorem that $L$ has a faithful finite-dimensional completely reducible representation. We establish the result that $C$, the center of $A$, is a finitely generated ring extension of $K$, and deter mine the degree of transcendence of the quotient field of $C$ over $K$. Zassenhaus has announced [15] a precise determination of the bound

Presented to the Society, December 28, 1952; received by the editors September 2, 1952 and, in revised form, April 25, 1953.

${ }^{1}$ Numbers in brackets refer to the references at the end of the paper. 
on the degrees of the absolutely irreducible representations of $A$. We give in $\$ 6$ an example due to $\mathrm{N}$. Jacobson of a universal associative algebra of a two-dimensional Lie algebra over a field of characteristic zero which is primitive, and therefore has an infinite-dimensional irreducible representation.

2. Definitions and preliminary results. Throughout the paper $S$ denotes a noncommutative ring with an identity element, and $R$ a subring of the center of $S$ containing the identity element; we shall then call $S$ an extension of $R$. We shall assume frequently that $S$ is a finite extension of $R$ in the sense that $S$ is a finitely generated $R$ module, or that $S$ is an integral extension, which means that $S$ satisfies the ascending chain condition (A.C.C.) for $R$-submodules. Every integral extension is a finite extension, and if $R$ is a Noetherian ring, any finite extension of $R$ is an integral extension. If $z$ is an element of an integral extension of $R$, then the powers of $z$ generate a finite $R$ submodule, and there exists a relation

$$
z^{n}+a_{n-1} z^{n-1}+\cdots+a_{0}=0, \quad a_{i} \in R .
$$

An extension in which every element satisfies an equation of the form (1) is not necessarily an integral extension (since there exist infinitedimensional algebraic algebras, for instance), and we shall establish some of our results for extensions of this type, although the particular extensions to which we apply our results are integral extensions.

A representation of $S$ is a homomorphism of $S$ onto a subring $S^{\prime}$ of the ring of endomorphisms of a commutative group; the representation is irreducible if the group has no proper $S^{\prime}$-submodules. If $R$ is an extension of a field $K$, then any representation of $S$ maps $K$ isomorphically onto a subfield $K^{\prime}$ of $S^{\prime}$, and $S^{\prime}$ is an algebra over $K^{\prime}$. We say that the representation is finite or algebraic over $K$ if $S^{\prime}$ is a finite-dimensional or algebraic algebra, respectively, over $K^{\prime}$. If the representation is irreducible, and if the identity element of $K^{\prime}$ is the identity operator on this group, then the representation is finite over $K$ if and only if the representation space is finite-dimensional over $K^{\prime}$. If $S$ is an extension of a field $K$, then every homomorphic image of $S$ contains a subfield isomorphic to $K$, and we shall identify $K$ with the subfields obtained in this way; thus if $\mathfrak{U}$ is an ideal in $S, S / \mathfrak{U}$ is an algebra over $K$.

By $S[X]$ or $S\left[X_{1}, \cdots, X_{n}\right]$ we mean the ring of polynomials in one or $n$ variables respectively, where we assume that the variables are algebraically independent, and are commutative with each other and with the elements of $S$. If $S$ is a subring of a ring $T$, and if $u_{1}, \cdots, u_{n}$ are mutually commutative elements of $T$, which also 
commute with the elements of $S$, then $S\left[u_{1}, \cdots, u_{n}\right]$ means the subring of $T$ generated by $S$ and the elements $u_{i} ; S\left[u_{1}, \cdots, u_{n}\right]$ is a homomorphic image of $S\left[X_{1}, \cdots, X_{n}\right]$.

According to Goldman [6], a commutative ring $R$ with an identity element is a Hilbert ring if every prime ideal in $R$ is an intersection of maximal ideals, that is, in every homomorphic image of $R$, the Jacobson radical and the nil radical coincide. We list for future reference the following facts about Hilbert rings, proofs of which have been given by Goldman [6] and Krull [11].

2.1. If $R$ is a Hilbert ring, then every homomorphic image of $R$ is a Hilbert ring.

2.2. $R$ is a Hilbert ring if and only if $R[X]$ is a Hilbert ring.

2.3. Let $R$ be a Hilbert ring. If $\mathfrak{M}$ is a maximal ideal in $R[X]$, then $R \cap \mathfrak{M}$ is a maximal ideal in $R$, and $R[X] / \mathfrak{M}$ is an algebraic extension of the field $R / R \cap \mathfrak{M}$.

2.4. If $K$ is a field, and if $m$ is a maximal ideal in $K\left[X_{1}, \cdots, X_{n}\right]$, then $K\left[X_{1}, \cdots, X_{n}\right] / \mathfrak{m}$ is algebraic over $K$.

In order to explain what significance these theorems have in the present context, we consider first the connection between irreducible representations and primitive ideals. An ideal $\mathfrak{U}$ in an arbitrary ring $S$ is primitive if the ring $S / \mathfrak{U}$ is a primitive ring [9, Definition 3]; thus $\mathfrak{U}$ is primitive if and only if $\mathfrak{U}$ is the kernel of an irreducible representation of $S$ [9, Theorem 21]. If $S$ is a commutative, with an identity element, then $\mathfrak{U}$ is primitive if and only if it is maximal. It is known [9, Theorem 20] that the Jacobson radical is the intersection of the primitive ideals in the ring, and it follows that the intersection of the primitive ideals containing an ideal $\mathscr{B}$ is the ideal which maps onto the Jacobson radical of $S / \mathfrak{B}$.

Levitzki has proved [13] that the intersection of the prime ideals in a ring ( $\mathfrak{B}$ is prime if, whenever $\mathscr{A}$ and $\mathfrak{B}$ are ideals, $\mathscr{A} \mathscr{B} \subseteq \mathfrak{B}$ implies $\mathfrak{A} \subseteq \mathfrak{B}$ or $\mathfrak{B} \subseteq \mathfrak{B}$ ) is the lower radical in the sense of Baer [2]. Therefore the statement that in $S$ every prime ideal is an intersection of primitive ideals is equivalent to the statement that in every homomorphic image of $S$ the Jacobson radical coincides with the lower radical, and the commutative rings with this property are precisely the Hilbert rings.

\section{Prime and primitive ideals in finite extensions.}

LeMma 3.1. Let $S$ be an extension of $R$. If every element of $S$ satisfies an equation of the form (1), then every primitive ideal in $S$ contracts to a maximal ideal in $R$.

Proof. Let $\mathfrak{U}$ be a primitive ideal in $S$; we have to prove that 
$\mathfrak{u} \cap R$ is a maximal ideal in $R$. Since $\mathfrak{U}$ is primitive, $\mathfrak{U}$ is prime in $S[9$, p. 316] and this implies that $\mathfrak{U} \cap R=\mathfrak{u}$ is prime in $R$; in fact, if $a b \in \mathfrak{u}, a, b \in R$, then $a S b=a b S \subseteq \mathfrak{U}$, and hence $a \in \mathfrak{U} \cap R$ or $b \in \mathfrak{U} \cap R$. Now $R / \mathfrak{u}=R^{*}$ is an integral domain isomorphic to a subring of the center of the primitive ring $S^{*}=S / \mathfrak{u}$, and we shall identify $R^{*}$ with this subring. $S^{*}$ in turn may be identified with an irreducible ring of endomorphisms acting in a commutative group $G$, and $G$ is cyclic relative to $S^{*}$, that is, $G=u S^{*}$ for some $u \in G$. Let $\Im$ be the right ideal in $S^{*}$ of elements $a$ such that $u a=0$, and let $B$ be the subring of $S^{*}$ consisting of elements $b$ such that $b \Im \subseteq \Im$. Then $D=B / \Im$ is antiisomorphic to the centralizer of $S^{*}$ in the ring of endomorphisms of $G$, and is a division ring $[8, \mathrm{p} .236]$. On the other hand $R^{*} \subseteq B$, and $R^{*} \cap \Im=0$, so that $R^{*}$ is isomorphic to a subring of $D$, which we shall again identify with $R^{*}$. Let $a^{*}$ be a nonzero element of $R^{*}$; then $a^{*}$ has an inverse $z^{*}$ in $D$. Let $\pi$ and $\rho$ be the natural mappings of $S$ onto $S^{*}$ and $B$ onto $D$ respectively. Choose $z \in S$ such that $z \pi \rho=z^{*}$; then $z$ satisfies an equation of the form (1), and if we apply the mapping $\pi \rho$ to this equation we obtain

$$
\left(a^{*}\right)^{-n}+r_{n-1}^{*}\left(a^{*}\right)^{-n+1}+\cdots+r_{0}^{*}=0, \quad{ }_{r_{i}}^{*} \in R^{*},
$$

where the $r_{i}^{*}$ are not all zero since $a^{*-1}$ is not nilpotent. Then we have

$$
\left(a^{*}\right)^{-1}=-r_{n-1}^{*}-\cdots-r_{0}^{*}\left(a^{*}\right)^{n-1} \in R^{*}
$$

so that $R^{*}$ is a field and $\mathfrak{u} \cap R$ a maximal ideal.

COROLlARY. Let $S$ be a primitive ring with an identity element such that every element satisfies an equation of the form (1) with coefficients in the center. Then the center of $S$ is a field.

We remark that it is not true in general that the center of a primitive ring with an identity element is a field. Indeed, consider the ring $S$ of row finite matrices $\left(a_{i j}\right)$ with rational coefficients $a_{i j}$ such that $a_{i j}=\delta_{i j} a$ for sufficiently large $i$ and $j$, where $a$ is an integer. Then $S$ is isomorphic to a dense ring of linear transformations in a vector space of countable dimension over the field of rational numbers, and hence is a primitive ring, but it is easy to see that the center of $S$ is isomorphic to the ring of integers, which is not a field.

LEMMA 3.2. Let $S$ be an integral extension of $R$. If $\mathfrak{p}$ is a prime ideal in $R$, then there exists a prime ideal $\mathfrak{P}$ in $S$ such that $\mathfrak{P} \cap R=\mathfrak{p}$.

Proof. If $Z$ is the set of ideals in $S$ which contract to ideals contained in $\mathfrak{p}$, then $Z$ is not vacuous, and by Zorn's Lemma $Z$ contains 
a maximal element $\mathfrak{B}$. By making heavy use of the fact that $R$ is contained in the center of $S$, the argument given by Cohen and Seidenberg [4, Theorem 2] for the commutative case can be applied to show that $\mathfrak{B}$ is prime and that $\mathfrak{B} \cap R=\mathfrak{p}$.

Lemma 3.3. Let $S$ be a finite extension of $R$, and let $\mathfrak{p}, \mathfrak{B}$ be prime ideals in $R$ and $S$ respectively such that $\mathfrak{B} \cap R=\mathfrak{p}$. If $\mathfrak{p}$ is maximal then $\mathfrak{B}$ is primitive.

Proof. The ring $S / \mathfrak{P}$ is a finite module over the field $R / \mathfrak{p}$, and consequently satisfies the minimum condition for right ideals. By a well known argument (cf. $[5$, p. 73]) the fact that the zero ideal of $S / \mathfrak{B}$ is prime implies that $S / \mathfrak{P}$ is a simple ring, and hence the ideal $\mathfrak{B}$ is primitive, since. $S$ contains an identity element.

\section{The irreducible representations.}

THeOREM 4.1. Let $S$ be an integral extension of its center $R$. If $T$ is a primitive homomorphic image of $S$, then the center $K$ of $T$ is a field, and $T$ is finite over $K$.

We shall give two proofs of this result, for the second of which we are indebted to N. Jacobson.

FIrst PROOF. Since $S$ satisfies the A.C.C. for $R$-submodules, $T$ satisfies the A.C.C. for $K$-submodules, and hence, by the corollary to Lemma $3.1, K$ is a field. But a vector space over a field $K$ which satisfies the A.C.C. for $K$-submodules is necessarily finite-dimensional over $K$, and the theorem is proved.

SECOND PROOF. As in the beginning of the proof of Lemma 3.1 it follows that the center of $T$ is an integral domain, and $T$ is a finite $K$-module, by the hypothesis of the theorem. If we imbed $T$ in the ring of quotients $a b^{-1}, a \in T, b \in K, b \neq 0$, which can be done since no element of $K$ can be a zero divisor in $T$ (for $a b=0, a \in K$ implies $T(a b)=a T b=0$ whence $a=0$ or $b=0$ ) we obtain an algebra $A$, finitedimensional over the quotient field $L$ of $K$. Such an algebra satisfies a polynomial identity with coefficients \pm 1 , which is also satisfied by its subring $T$. By Kaplansky's theorem ${ }^{2} K$ is a field and $T$ is finite over $K$.

THEOREM 4.2. Let $S$ be an integral extension of $R$, and let $R$ be an extension of a field $E$ such that if $\mathfrak{m}$ is a maximal ideal in $R$ then ${ }^{3}$

2 Cf. I. Kaplansky, Rings with a polynomial identity, Bull. Amer. Math. Soc. vol. 54 , pp. 575-580, particularly Theorem 1 and (c) on p. 580.

3 We shall use the notation $(R / \mathrm{m}: E)$ for the dimension over the field $E$ of the algebra $R / \mathfrak{m}$. 
$(R / \mathfrak{m}: E)$ is finite. Then every irreducible representation of $S$ is finite over $E$.

Proof. Let $\mathfrak{U}$ be a primitive ideal in $S$. It is sufficient to prove that $(S / \mathfrak{u}: E)$ is finite. By Lemma 3.1, $\mathfrak{u} \cap R$ is a maximal ideal in $R$, and $(S / \mathfrak{U}: R / \mathfrak{u} \cap R)$ is finite since $S / \mathfrak{u}$ is a finite module over the field $R / \mathfrak{u} \cap R$. The argument is completed by the observation that

$$
(S / \mathfrak{u}: E)=(S / \mathfrak{u}: R / \mathfrak{u} \cap R)(R / \mathfrak{u} \cap R: E)
$$

and $(R / \mathfrak{u} \cap R: E)$ is finite by hypothesis.

CoROllary 1. Let $R$ satisfy the hypothesis of the theorem. If $N$ is the minimum number of generators of the module $S$ over $R$, then the degree 4 of every absolutely irreducible representation of $S$ does not exceed $N^{1 / 2}$.

Proof. Let $\sigma: S \rightarrow S^{\sigma}$ be an absolutely irreducible representation of $S$, with kernel $\mathfrak{U}$, and representation space $V$. By Theorem 4.2, $S^{\sigma}$ is a finite-dimensional simple subalgebra of the algebra of linear transformations of $V$ over $E$, and it follows from Burnside's Theorem that $S^{\sigma}$ is the full algebra of linear transformations in $V$ over $E$. Therefore the degree of $\sigma$ does not exceed $\left(S^{\sigma}: E\right)^{1 / 2}$. By (2) the latter does not exceed $N^{1 / 2}(R / \mathfrak{U} \cap R: E)^{1 / 2}$, and we prove now that $(R / R \cap \mathfrak{U l}: E)=1$. By hypothesis the algebra $S_{K}^{\sigma}$ obtained by extending the field $E$ to $K$ is primitive for arbitrary extension fields $K$. On the other hand it follows easily from the properties of Kronecker products that every $(R / \mathfrak{U} \cap R)_{K}$-submodule of $(S / \mathfrak{u})_{K}$ is finitely generated, and an application of Lemma 3.1 shows that $(R / \mathfrak{U} \cap R)_{K}$ is a field for arbitrary $K$, finite over $K$ since $(R / \mathfrak{U} \cap R: E)=\left((R / \mathfrak{u} \cap R)_{K}: K\right)$. If now we let $K$ be the algebraic closure of $E$, then $\left((R / \mathfrak{u} \cap R)_{K}: K\right)=1$, and $(R / \mathfrak{u} \cap R: E)=1$ as required.

COROLLARY 2. Let $R$ satisfy the hypothesis of the theorem. If $\sigma_{1}$ and $\sigma_{2}$ are irreducible representations of $S$ with representation modules $V_{1}$ and $V_{2}$ respectively, then $\sigma_{1}$ and $\sigma_{2}$ are equivalent, that is, $V_{1}$ and $V_{2}$ are operator isomorphic if and only if the kernels of $\sigma_{1}$ and $\sigma_{2}$ coincide.

Proof. By Theorem 4.2, if $\sigma_{1}: S \rightarrow S_{1}$ and $\sigma_{2}: S \rightarrow S_{2}$, then $S_{1}$ and $S_{2}$ are finite-dimensional simple algebras over $E$, both faithfully represented on the corresponding representation spaces. Then each $V_{i}$ is operator isomorphic to a minimal right ideal in $S_{i}$, and these in turn are isomorphic to each other if and only if $S_{1}$ is isomorphic to $S_{2}$, but

4 Here we regard $S$ as an algebra over the field $E$, so that the degree of a representation of $S$ refers to the dimension over $E$ of the corresponding representation space. 
this is precisely the condition that the kernels of $\sigma_{1}$ and $\sigma_{2}$ coincide.

TheOREM 4.3. Let $S$ be an integral extension of $R$. Every prime ideal in $S$ is an intersection of primitive ideals if and only if $R$ is a Hilbert ring.

Proof. First we prove that the condition is necessary. Let $\mathfrak{p}$ be a prime ideal in $R$; we have to prove that $\mathfrak{p}$ is an intersection of maximal ideals, and to accomplish this, it is sufficient to show that if $a$ is an arbitrary element of $R$ not in $\mathfrak{p}$, then there is a maximal ideal containing $\mathfrak{p}$ but not $a$. By Lemma 3.2 there is a prime ideal $\mathfrak{B}$ in $S$ such that $\mathfrak{B} \cap R=\mathfrak{p}$. Then $a \notin \mathfrak{B}$, and there exists a primitive ideal $\mathfrak{u}$ containing $\mathfrak{B}$ but not $a$. By Lemma 3.1, $\mathfrak{U} \cap R$ is a maximal ideal with the required properties.

Conversely, let $R$ be a Hilbert ring. We shall prove that if $\mathfrak{P}$ is a prime ideal in $S$ then the Jacobson radical $\mathfrak{R}$ of $S / \mathfrak{B}$ is nilpotent, and hence the zero ideal since $\mathfrak{B}$ is prime. The fact that $S$ is an integral extension of $R$ implies that $S$ satisfies the A.C.C. for both right and left ideals, and by a result of Levitzki [12], $\mathfrak{N}$ will be nilpotent if we can prove that $\mathfrak{N}$ is a nil ideal. Now the residue class ring $S / \mathfrak{P}$ is an integral extension of $R_{1}=R / \mathfrak{P} \cap R$, and by [1, Theorem 9], if $z$ is an element of $\Re$, then the quasi inverse of $z$ is in the ring $R_{1}[z]$, and therefore $z$ is in the Jacobson radical of $R_{1}[z]$. On the other hand $R_{1}[z]$ is a homomorphic image of $R_{1}[X]$, and is a Hilbert ring by 2.1 and 2.2. Then $z$ is in the intersection of all the prime ideals of $R_{1}[z]$, and hence is nilpotent.

As one illustration of these methods we prove two theorems about polynomial rings.

THEOREM 4.4. Let $S$ be an integral extension of $R$. Every prime ideal in $S$ is an intersection of primitive ideals if and only if the same condition is valid for $S[X]$.

Proof. By an argument similar to the proof of the Hilbert basis theorem, it can be shown that $S[X]$ is an integral extension of $R[X]$. Then by Theorem 4.3 and 2.2 , the following statements are equivalent: (i) every prime ideal in $S$ is an intersection of primitive ideals, (ii) $R$ is a Hilbert ring, (iii) $R[X]$ is a Hilbert ring, and (iv) every prime ideal in $S[X]$ is an intersection of primitive ideals. This completes the proof.

THEOREM 4.5. Let $S$ be an integral extension of $R$, in which every prime ideal is an intersection of primitive ideals. Let $R$ contain a field $K$ such that every irreducible representation of $S$ is finite over $K$. Then every irreducible representation of $S[X]$ is finite over $K$. 
Proof. In the proof of Theorem 4.4 we have shown that $S[X]$ is an integral extension of $R[X]$. The present result will follow from Theorem 4.2 if we can prove that every field homomorphic to $R[X]$ is finite over $K$. First we observe that if $\mathfrak{m}$ is a maximal ideal in $R$, then by Lemmas 3.2 and 3.3 there exists a primitive ideal $\mathfrak{M}$ in $S$ such that $\mathfrak{M} \cap R=\mathfrak{m}$. The field $R / \mathfrak{m}$ is a $K$-subspace of the finite-dimensional algebra $S / \mathfrak{M}$, and consequently $R / \mathfrak{m}$ is finite over $K$. By Theorem 4.3, $R$ is a Hilbert ring, and finally by 2.3 we see that if $\mathfrak{U}$ is maximal in $R[X]$, then $(R[X] / \mathfrak{U}: K)$ is finite.

The last two theorems afford some insight toward the ideal theory of a particular simple type of ring extension; thus information about ideals or irreducible representations of a ring $S$ is passed on to rings of the form $S\left[X_{1}, \cdots, X_{n}\right]$ and their homomorphic images.

5. Application to universal associative algebras of Lie algebras of characteristic $p \neq 0$. Let $L$ be a finite-dimensional Lie algebra over a field $K$ of prime characteristic. If $u_{1}, \cdots, u_{n}$ is a basis for $L$ over $K$, then the universal associative algebra $A$ of $L$ (cf. $[3 ; 7 ; 14])$ has a basis consisting of the standard monomials $u_{1}^{e_{1}} \cdots u_{n}^{e_{n}}, e_{i} \geqq 0$. Although for some purposes it is sufficient to consider the subalgebra of $A$ consisting of elements with zero constant term, we shall find it convenient to assume that $A$ has an identity element, and hence contains $K$ as a subfield. Jacobson has proved in [10] the following facts: (i) for each $i, 1 \leqq i \leqq n$, there exists a nonzero polynomial $f_{i}(X)$ such that $y_{i}=f_{i}\left(u_{i}\right)$ is in the center of $A$; and (ii) every element of $A$ can be expressed as a linear combination of terms of the form $y_{1}^{m_{1}} \cdots y_{n}^{m_{n}} u_{1}^{\lambda_{1}} \cdots u_{n}^{\lambda_{n}}, m_{i} \geqq 0$, and $0 \leqq \lambda_{i}<d_{i}=\operatorname{deg} f_{i}(X)$. Let $R$ $=K\left[y_{1}, \cdots, y_{n}\right]$. Then $R$ is a Noetherian Hilbert ring, since $R$ is a homomorphic image ${ }^{5}$ of $K\left[X_{1}, \cdots, X_{n}\right]$, and by 2.4 , it follows that every field which is a homomorphic image of $R$ is finite over $K$. By statement (ii) we see that $A$ is a finite extension of $R$, and since $R$ is Noetherian, $A$ is actually an integral extension of $R$. Theorems 4.2 and 4.3 , together with their corollaries, can now be applied to $R$. As we pointed out earlier, the finiteness of the degrees of the irreducible representations can also be established by observing that $A$ satisfies a polynomial identity. We summarize these observations in the following theorem.

THEOREM 5.1. Every prime ideal in $A$ is an intersection of primitive ideals. If $\mathfrak{U}$ is a primitive ideal in $A$, then $A / \mathfrak{U}$ is finite over $K$. The degree of every absolutely irreducible representation of $A$ does not exceed

\footnotetext{
${ }^{5}$ We shall prove later in this section that the $y_{i}$ are algebraically independent over
} $K$, so that $R$ is actually isomorphic to $K\left[X_{1}, \cdots, X_{n}\right]$. 
$N^{1 / 2}$, where $N$ is the product of the degrees of the polynomials $f_{i}(X)$.

CoRollary 1. A has sufficiently many finite-dimensional irreducible representations, that is, for each $z \neq 0$ in $A$ there exists a finite dimensional irreducible representation $\sigma$ such that $z^{\sigma} \neq 0$.

Proof. It follows from the fact that the units of $A$ are the nonzero elements of $K$ that $A$ is semi-simple (cf. [9, p. 303]). Therefore the intersection of the primitive ideals is the zero ideal, and since each primitive ideal is the kernel of a finite-dimensional irreducible representation, the proof is complete.

Corollary 2. Let $\mathfrak{B}$ be an ideal in $A$. Let $z$ be an element of $A$ which is sent into zero by every irreducible representation which annihilates $\mathfrak{B}$. Then $z^{r} \in \mathfrak{B}$ for some positive integer $r$.

Proof. By hypothesis $z$ is contained in the intersection of all the primitive ideals containing $\mathfrak{B}$. From the first statement of Theorem 5.1 it follows that $z$ is also contained in the ideal which maps onto the lower radical of $A / \mathscr{B}$. Since the lower radical is a nil ideal, we have established the result.

The following application of Corollary 1 was pointed out to me by $\mathrm{N}$. Jacobson, and was attributed by him to M. Lazard.

THEOREM 5.2. If $L$ is a finite-dimensional Lie algebra over a field of prime characteristic, then $L$ has a faithful completely reducible representation on a finite-dimensional space.

Proof. We may identify $L$ with the linear part of $A$. If $u_{1}, \cdots, u_{n}$ is a basis of $L$, then by Corollary 1 , for each $i, 1 \leqq i \leqq n$, there exists a primitive ideal $\mathfrak{U}_{i}$ which does not contain $u_{i}$, and such that $A / \mathfrak{U}_{i}$ is finite over $K$. Since no $u_{i}$ is in $\mathfrak{U}=\cap \mathfrak{U}_{i}, L$ is imbedded isomorphically in $A / \mathfrak{U}$.If $\mathfrak{u}_{1}, \cdots, \mathfrak{U}_{r}, r \leqq n$, are the distinct $\mathfrak{U}_{i}$, then these ideals are relatively prime in pairs; in fact if $\mathfrak{U}_{i}+\mathfrak{U}_{j}, i \neq j$, is properly contained in $A$, then $\mathfrak{U}_{i}+\mathfrak{U}_{j}$, and hence $\mathfrak{U}_{i}$ and $\mathfrak{u}_{j}$ separately, are contained in a primitive ideal $\mathfrak{W}$. But $A / \mathfrak{U}_{i}$, for example, is a finite-dimensional simple algebra, so that the kernel of the natural homomorphism of $A / \mathfrak{U}_{i}$ onto $A / \mathfrak{W}$, which is $\mathfrak{W} / \mathfrak{U}_{i}$, is the zero ideal in $A / \mathfrak{U}_{i}$, and $\mathfrak{W}=\mathfrak{U}_{i}$. Similarly $\mathfrak{W}=\mathfrak{U}_{j}$, but this is impossible if $\mathfrak{u}_{i}$ and $\mathfrak{u}_{j}$ are distinct. By the Chinese Remainder Theorem, $A / \mathfrak{U}$ is isomorphic to the direct sum $A / \mathfrak{U}_{1} \oplus \cdots \oplus A / \mathfrak{u}_{r}$, which is a finite-dimensional semisimple algebra containing $L$. Since $L$ generates $A / \mathfrak{U}_{1} \oplus \cdots \oplus A / \mathfrak{U}_{r}$, the fact that the semi-simple algebra has a faithful finite-dimensional completely reducible representation implies that $L$ has one also.

The ring $R=K\left[y_{1}, \cdots, y_{n}\right]$ which has played an essential role in 
our discussion could have been replaced by the center $C$ of $A$. In fact, the center $C$ is a finite $R$-module, so that $C=w_{1} R+\cdots+w_{t} R$; then $C=K\left[y_{1}, \cdots, y_{n}, w_{1}, \cdots, w_{t}\right]$, and $C$ also is a Noetherian Hilbert ring, and a finitely generated ring extension of $K$. Since $C$ is integrally dependent on $R$, the degree of transcendence of the quotient field of $C$ is not greater than $n$, the dimension of $L$ over $K$. We shall indicate a proof of the fact that the degree of transcendence of $C$ over $K$ is exactly $n$. It will be sufficient to prove that the generators $y_{1}, \cdots, y_{n}$ of $R$ are algebraically independent over $K$. Since each $y_{i}=f_{i}\left(u_{i}\right)$, the $y_{i}$ are distinct and linearly independent. Suppose we have a relation

$$
\alpha_{0}+\sum \alpha_{(i)} y_{1}^{i_{1}} \cdots y_{n}^{i_{n}}=0, \quad(i)=\left(i_{1}, \cdots, i_{n}\right) \neq 0,
$$

where the power products of the $y_{i}$ are distinct. If not all of the coefficients are zero, choose a term $\alpha_{(k)} y_{1}^{k_{1}} \ldots y_{n}^{k_{n}}$ of maximum degree, whose coefficient is different from zero. Then we have

$$
-\alpha_{(k)} y_{1}^{k_{1}} \cdots y_{n}^{k_{n}}=\alpha_{0}+\sum_{(i) \neq(k)} \alpha_{(i)} y_{1}^{i_{1}} \cdots y_{n}^{i_{n}}
$$

Now if in (3) we substitute $y_{i}=f_{i}\left(u_{i}\right)$ we then obtain on the left side a monomial with nonzero coefficient of the form

$$
\eta u_{1}^{d_{1} k_{1}} \ldots u_{n}^{d_{n} k_{n}}, \quad \eta \in K, d_{i}=\operatorname{deg} f_{i} .
$$

But no monomial of this form can appear on the right side, because the only possibilities are the terms of maximum degree $\sum d_{i} k_{i}$ in the $u_{i}$, and if we examine a term on the right side, say $\xi u_{1}^{d_{1} i_{1}} \cdots u_{n}^{d_{n} t_{n}}$, $\xi \in K$, we see that some exponent $d_{j} i_{j}$ is different from the corresponding $d_{j} k_{j}$, otherwise $(i)=(k)$, contrary to assumption. But since the standard monomials are linearly independent, a relation of the form (3) is impossible, and we are forced to conclude that all the coefficients of the original relation are zero.

6. An example. It follows from Theorem 5.1 that no universal associative algebra of a Lie algebra over a field of prime characteristic can be a primitive algebra. We give now an example, which was presented by $\mathrm{N}$. Jacobson in his course at Yale in 1949-50, of a Lie algebra over a field of characteristic zero whose universal associative algebra is primitive. Let $K$ be a field of characteristic zero, and let $L$ be the solvable Lie algebra over $K$ with basis elements $x$ and $y$ satisfying the commutation rules $[x, y]=-[y, x]=x$. Let $A$ be the universal associative algebra of $L$. In order to show that $A$ is primitive it is sufficient to find a maximal right ideal $\mathfrak{U}$ in $A$ such that $(\mathfrak{U}: A)=0$ (cf. $[9$, p. 312]). Since the element $x+1$ does not have a right inverse, 
the right ideal $(x+1) A$ does not coincide with $A$, and is contained in a maximal right ideal $\mathfrak{u}$. We prove that $(\mathfrak{u}: A)=0$. Since $(\mathfrak{u}: A)$ is a two-sided ideal, $[x, z]=x \mathfrak{s}-z x \in(\mathfrak{u}: A), z \in(\mathfrak{U}: A)$. The formulae $x \phi(y)=\phi(y+1) x, \quad[x, \phi(y)]=(\phi(y+1)-\phi(y)) x$, and $\left[x, x^{r} \phi(y)\right]$ $=x^{r}(\phi(y+1)-\phi(y)) x$, which are valid for arbitrary $\phi(y) \in K[y]$ and all positive integers $r$, enable us to show, since $\phi(y+1)-\phi(y)$ has lower degree than $\phi(y)$, that if $(\mathfrak{u}: A)$ is not the zero ideal, then a nonzero polynomial in $x, \psi(x) \in(\mathfrak{U}: A)$. Then upon using the rule $\left[x^{n}, y\right]=n x^{n}$, we can prove that a power of $x$, say $x^{\circ}$, is in $(\mathfrak{U}: A)$. It follows that $x^{2^{m}} \in \mathfrak{U}$ for some positive integer $m$, but on the other hand, since $x+1 \in \mathfrak{U},(x+1)(x-1)=x^{2}-1, \cdots, x^{2^{m}}-1 \in \mathfrak{U}$, and we have $1 \in \mathfrak{U}$. Our assumption that $(\mathfrak{u}: A) \neq 0$ is thus untenable, and we conclude that $A$ is primitive.

\section{REFERENCES}

1. G. Azumaya, On maximally central algebras, Nagoya Math. J. vol. 2 (1951) pp. 119-150.

2. R. Baer, Radical ideals, Amer. J. Math. vol. 65 (1943) pp. 537-568.

3. G. Birkhoff, Representability of Lie algebras and Lie groups by matrices, Ann. of Math. vol. 38 (1937) pp. 526-532.

4. I. S. Cohen and A. Seidenberg, Prime ideals and integral dependence, Bull. Amer. Math. Soc. vol. 52 (1946) pp. 252-261.

5. M. Deuring, Algebren, Berlin, 1935.

6. O. Goldman, Hilbert rings and the Hilbert Nullstellensatz, Math. Zeit. vol. 54 (1951) pp. 136-140.

7. Harish-Chandra, On representations of Lie algebras, Ann. of Math. vol. 50 (1949) pp. 900-915.

8. N. Jacobson, Structure theory of simple rings without finileness assumptions, Trans. Amer. Math. Soc. vol. 57 (1945) pp. 228-245.

9. - The radical and semi-simplicily for arbitrary rings, Amer. J. Math. vol. 67 (1945) pp. 300-320.

10. - A note on Lie algebras of characteristic p, Amer. J. Math. vol. 74 (1952) pp. $357-359$.

11. W. Krull, Jacobsonsche Ringe, Hilbertscher Nullstellensatz, Dimensionstheorie, Math. Zeit. vol. 54 (1951) pp. 354-357. 80.

12. J. Levitzki, On multiplicative systems, Compositio Math. vol. 8 (1950) pp. 76-

13. - Prime ideals and the lower radical, Amer. J. Math. vol. 73 (1951) pp. 25-29.

14. E. Witt, Treue Darstellung Liescher Ringe, J. Reine Angew. Math. vol 177 (1937) pp. 152-160.

15. H. Zassenhaus, On the representations of Lie algebras of prime characteristic, Bull. Amer. Math. Soc. Abstract 59-1-7.

UNIVERSITY OF WISCONSIN 